\title{
Muscle overexpression of Klf15 via an AAV8-Spc5-12 construct does not provide benefits in spinal muscular atrophy mice
}

\author{
Nina Ahlskog ${ }^{1,2} \cdot$ Daniel Hayler $^{3} \cdot$ Anja Krueger $^{1} \cdot$ Sabrina Kubinski $^{4,5} \cdot$ Peter Claus $^{4,5} \cdot$ Suzan M. Hammond ${ }^{2,6}$. \\ Matthew J. A. Wood ${ }^{2,6} \cdot$ Rafael J. Yáñez-Muñoz ${ }^{3} \cdot$ Melissa Bowerman (iD ${ }^{1,7,8}$
}

Received: 28 July 2019 / Revised: 19 March 2020 / Accepted: 27 March 2020 / Published online: 20 April 2020

(c) The Author(s) 2020. This article is published with open access

\begin{abstract}
Spinal muscular atrophy (SMA) is a neuromuscular disease caused by loss of the survival motor neuron (SMN) gene. While there are currently two approved gene-based therapies for SMA, availability, high cost, and differences in patient response indicate that alternative treatment options are needed. Optimal therapeutic strategies will likely be a combination of SMN-dependent and -independent treatments aimed at alleviating symptoms in the central nervous system and peripheral muscles. Krüppel-like factor 15 (KLF15) is a transcription factor that regulates key metabolic and ergogenic pathways in muscle. We have recently reported significant downregulation of Klf15 in muscle of presymptomatic SMA mice. Importantly, perinatal upregulation of Klf15 via transgenic and pharmacological methods resulted in improved disease phenotypes in SMA mice, including weight and survival. In the current study, we designed an adeno-associated virus serotype 8 (AAV8) vector to overexpress a codon-optimized Klf15 cDNA under the muscle-specific Spc5-12 promoter (AAV8-Klf15). Administration of AAV8-Klf15 to severe Taiwanese Smn ${ }^{-1-}$; $S M N 2$ or intermediate $S m n^{2 B /-}$ SMA mice significantly increased Klf15 expression in muscle. We also observed significant activity of the AAV8-Klf15 vector in liver and heart. AAV8-mediated Klf15 overexpression moderately improved survival in the $S m n^{2 B /-}$ model but not in the Taiwanese mice. An inability to specifically induce Klf15 expression at physiological levels in a time- and tissue-dependent manner may have contributed to this limited efficacy. Thus, our work demonstrates that an AAV8Spc5-12 vector induces high gene expression as early as P2 in several tissues including muscle, heart, and liver, but highlights the challenges of achieving meaningful vector-mediated transgene expression of Klf 15.
\end{abstract}

\section{Introduction}

Spinal muscular atrophy (SMA) is a devastating childhood neuromuscular disease that leads to early death in the most severe cases $[1,2]$. As an autosomal recessive disease, SMA is caused by loss of the survival motor neuron 1 (SMN1) gene

Supplementary information The online version of this article (https:// doi.org/10.1038/s41434-020-0146-8) contains supplementary material, which is available to authorized users.

Melissa Bowerman

m.bowerman@keele.ac.uk

1 Department of Physiology, Anatomy and Genetics, University of Oxford, Oxford, UK

2 Department of Paediatrics, University of Oxford, Oxford, UK

3 AGCTlab.org, Centre of Gene and Cell Therapy, Centre for Biomedical Sciences, Department of Biological Sciences, Royal Holloway University of London, Egham Hill, Egham, Surrey, UK due to either mutations or deletions [3]. While a total deficiency in the SMN protein is embryonic lethal [4], humans have a duplicated copy of SMN1, termed SMN2 [3], which allows for survival in the absence of the former. However, SMN2 contains a key $\mathrm{C}$ to $\mathrm{T}$ transition in exon 7 that leads to its excision in $\sim 90 \%$ of the transcripts produced, generating a non-functional SMN $\Delta 7$ protein that is rapidly degraded $[5,6]$. Importantly, the $10 \%$ of fully functional full length SMN protein produced from SMN2 is sufficient to allow survival, albeit not sufficient to prevent neuromuscular degeneration [7].

4 Institute of Neuroanatomy and Cell Biology, Hannover Medical School, Hannover, Germany

5 Center of Systems Neuroscience, Hannover, Germany

6 MDUK Oxford Neuromuscular Centre, University of Oxford, Oxford, UK

School of Medicine, Keele University, Staffordshire, UK

8 Wolfson Centre for Inherited Neuromuscular Disease, RJAH Orthopaedic Hospital, Oswestry, UK 
The first genetic therapy for SMA, nusinersen/Spinraza ${ }^{\mathrm{TM}}$, was approved in December 2016 by the Food and Drug Administration (FDA) and in June 2017 by the European Medicines Agency (EMA) [8]. This antisense oligonucleotide is delivered directly to the central nervous system (CNS) via a lumbar puncture and is aimed at promoting $S M N 2$ exon 7 inclusion [9]. Zolgensma ${ }^{\circledR}$ is a single, systemic of delivery of SMN1 via an adeno-associated virus serotype 9 (AAV9) gene therapy that received FDA approval in May 2019 [10]. Additional SMN-enhancing pharmacological compounds are also in the pipeline and anticipated to be approved for patient use in the near future [11]. While the benefits of these SMN-dependent drugs are undeniably remarkable, it is appreciated that they unfortunately do not represent a cure and will have to be supported by additional non-CNS and non-SMN therapeutic interventions to provide optimal care to all SMA patients [12-15].

Skeletal muscle with reduced levels of SMN displays both cell-autonomous and non-autonomous defects $[16,17]$ and is therefore an important therapeutic target for SMA. We have recently demonstrated the dysregulated expression of the transcription factor Krüppel-like factor 15 (Klf15) in skeletal muscle of SMA mice during disease progression [18]. KLF15 is crucial in the regulation of skeletal muscle metabolism and ergogenic properties [19-22]. Specifically, we observed a significant downregulation of Klf15 expression in presymptomatic SMA mice and found that its neonatal upregulation via pharmacological (prednisolone) or transgenic (muscle-specific Klf15 overexpression) interventions significantly improved several disease phenotypes in SMA mice [18]. However, prednisolone has pleiotropic activities and constitutive embryonic overexpression of Klfl5 in skeletal muscle of SMA may have resulted in compensatory mechanisms [23]. In this study, we thus set out to overexpress Klf15 in skeletal muscle of neonatal SMA mice via a self-complementary adeno-associated virus serotype $2 / 8$ and the Spc5-12 promoter. While this strategy led to substantial Klf15 expression in skeletal muscle of SMA mice and control littermates, there were no associated significant improvements in disease phenotypes. Nevertheless, AAV8-Klf15 injections resulted in pronounced expression as early as postnatal day 2 in several tissues, including muscle, liver, and heart, highlighting the potential of this specific viral construct for efficient perinatal delivery.

\section{Materials and methods}

\section{Animals}

Wild-type (WT) FVB/N mice were used for initial expression screening. The Taiwanese $\mathrm{Smn}^{-1-} ; S M N 2$
(FVB/N background, FVB.Cg-Smn1tm1HungTg(SMN2) 2Hung/J, RRID: J:59313) [24] and the $S m n^{2 B /-}$ [25] mice (generously provided by Dr Lyndsay M Murray, University of Edinburgh) were housed in individual ventilated cages (fed ad libitum, $12 \mathrm{~h}$ light: $12 \mathrm{~h}$ dark cycle) at the Biomedical Sciences Unit, University of Oxford, according to procedures authorized by the UK Home Office (Animal Scientific Procedures Act 1986). The viral constructs were diluted in sterile $0.9 \%$ saline and administered at the indicated dose at postnatal day (P) 0 by a facial vein intravenous injection [26]. Litters were randomly assigned to treatment at birth. For survival studies, animals were weighed daily and culled at indicated time points or upon reaching their defined humane endpoint as set out by the Home Office Project Licence. For the $S m n^{2 B /-}$ mice, weaned mice were given daily wet chow at the bottom of the cage to ensure proper access to food. Sample sizes were determined based on similar studies with SMA mice.

\section{ScAAV2/8-Spc5-12 constructs}

The generation of the self-complementary adeno-associated virus serotype 2/8 (scAAV2/8) vectors and quality control were performed by Atlantic Gene Therapies (Nantes, France). The synthetic Spc5-12 promoter [27] was used to drive the expression of eGFP or a codon-optimized Klfl5 sequence:

agcgcttcaccacaggctcggccaggccagcatggttgatcatctgctgectg tggacgagacattcagcagccctaagtgttctgtgggctacctgggcgacagact ggcctctagacagcettaccacatgctgecctctccaatcagcgaggacgactcc gatgtgtctagccettgtagctgtgcctctcctgacagccaggccttctgtagctgtt actctgctggacctggacctgaggctcagggctctatcctggatttcctgctgagca gagctacactcggctctggcggaggatctggcggaatcggagattcttctggecct gtgacatggggetcttggagaagggctagcgtgcccgtgaaagaggaacacttct gcttccetgagttcetgagcggcgacaccgatgacgtgtccagacctttccagcct acactggaagagatcgaagagttcctcgaagagaacatggaagccgaagtgaaa gaagcccetgagaacggctccegcgacctggaaacatgttctcagctgtctgccg gctctcacagaagccatctgcacctgaaagcgccggcagagagagatgtacac ctcctccaggtggaacatctggcggeggagcacaatctgctggcgaaggacetg ctcatgatggacctgtgcetgtgctgctgcaaatccagcetgtggctgtgaagcaa gaggctggaacaggaccagcttctcctggacaggctcctgaatctgtgaaggtgg cccagctgctggtcaacatccagggacaaacattcgccetgctgcctcaggtggt gcccagcagtaatctgaacctgcctagcaagttcgtgcggatcgctcctgtgccaa tcgctgctaagcctatcggatctggctctcttggtcctggaccagctggactgctcg tgggacagaagttccetaagaaccetgecgecgagctgctgaagatgcacaagt gtacattccceggctgctccaagatgtataccaagtcctctcacctgaaggcccac ctgagaaggcataccggcgagaagcctttcgcttgcacatggcctggatgtggct ggcggttcagcagatctgatgagctgagcaggcaccgcagatctcacagcggag tgaagccataccagtgtcetgtgtgcgagaagaagttcgccagaagcgaccacct gtccaagcacatcaaggtgcacagattccctagaagcagcagagccgtgcgggc catcaattgactgcagaagctt. 


\section{C2C12 cell line}

C2C12 myoblast cells [28] were maintained in growth media consisting of Dulbecco's Modified Eagle's Media (DMEM) supplemented with 10\% fetal bovine serum and 1\% Penicillin/Streptomycin (all Life Technologies). For AAV transduction experiments, growth media was changed to differentiation media consisting of DMEM, $2 \%$ horse serum, and 1\% Penicillin/Streptomycin (all Life Technologies). Cells were allowed to differentiate for 3 days, after which they were transduced with a MOI of $1 \mathrm{E} 10^{5}$. Cells were harvested 3 days post-transduction for molecular analyses (flow cytometry or qPCR, as described below).

\section{qPCR}

Quadriceps muscles, liver, and heart were harvested at the indicated time points during disease progression and immediately flash frozen. RNA was extracted with the RNeasy MiniKit (Qiagen). For C2C12 cells, the media was removed and cells were washed with phosphate buffered saline (PBS) before being directly lysed as per instructions within the RNeasy MiniKit (Qiagen). Reverse transcription was performed using the High-Capacity cDNA Reverse Transcription Kit (ThermoFisher Scientific). qPCR was performed using SYBR green Mastermix (ThermoFisher Scientific) and primers for the codon-optimized Klf15 sequence (Forward: AGGACCTGCTCATGATGGAC; Reverse: TGTTTGTCCCTGGATGTTGA). RNA polymerase II polypeptide $J$ (PolJ) was used as a validated stably expressed housekeeping gene [29] (Forward: ACCACACTCTGGGGAACATC; Reverse: CTCGCTG ATGAGGTCTGTGA). All primers were ordered from Integrated DNA Technologies.

\section{Viral quantification}

In order to establish virus penetration into the tissue, TaqMan (ThermoFisher Scientific) qPCRs were performed using two primer-probe sets (Integrated DNA Technologies) specific to the AAV8 capsid (1: Forward: GCTCTT CAACATCCAGGTCAA; Reverse: TGGTACTCCGAGT CCGTAAA; Probe: TGGTACTCCGAGTCCGTAAA; and 2: Forward: GACCACCTTCAACCAGTCAA; Reverse: CTGCAGCTCCCATTCAATTTC; Probe: TTCATCAC GCAATACAGCACCGGA). The results of the two primerprobe sets were each efficiency-adjusted and normalized to PolJ (Forward: GTGGTCTTCTTTGTTGATGGTG; Reverse: TTCGAGTCGTTCTTGCTCTTC; Probe: AAGC AGGCGTTGGGAACCTTAGT). The two primer-probe sets yielded similar results (data not shown) and only data with primer set 2 are shown. No significant difference was detected between $\mathrm{Smn}^{+/-} ; \mathrm{SMN} 2$ and $\mathrm{Smn}^{-/-}$;SMN2 mice for any of the tissues (data not shown) and mice from both genotypes were therefore pooled. No amplification could be seen in untreated samples (data not shown).

\section{Flow cytometry}

Differentiated $\mathrm{C} 2 \mathrm{C} 12$ cells 3 days post-transduction (AAV8-GFP, MOI of $1 \mathrm{E} 10^{5}$ ) and untreated cells were trypsinized and washed in fluorescence-activated cell sorting (FACS) buffer (PBS supplemented with $2 \%$ bovine serum albumin and $0.05 \%$ sodium azide). Cells were pelleted by centrifugation at $300 \times g$ for $5 \mathrm{~min}$. The final cell pellet was resuspended in $200 \mu$ l of FACS buffer and the cell suspension was further diluted 1:2 in FACS buffer before detection in the Cytek DxP8 flow cytometer $\left(\mathrm{Cytek}^{\circledR}\right.$ Biosciences). Cell viability was tested by adding $1 \mu \mathrm{l}$ Sytox Red (Thermofisher) to the final cell suspension for $5 \mathrm{~min}$ at RT. The gating strategy included gating around the Sytox Red (RedFL1 channel) negative population following FCS/ FCSW doublet exclusion. The remaining population was assessed for a GFP-shift by recording in the BluFL1 channel. A total of 10,000 cells was recorded for each sample replicate. Data were analyzed using Flowjo 10 software (TreeStar Inc.).

\section{Immunocytochemistry and immunohistochemistry}

$\mathrm{C} 2 \mathrm{C} 12$ cells transduced with the $e G F P$-expressing AAV vector were imaged live with a DM IRB microscope (Leica).

Quadriceps muscles were harvested at the indicated time points during disease progression, fixed in $4 \%$ paraformaldehyde, cryopreserved in $30 \%$ sucrose, and cryosectioned at a thickness of $12 \mu \mathrm{m}$. The sections were immunostained with chicken anti-GFP antibody (1:3000, Abcam, ab13970) and detected with Alexa-488-conjugated anti-chicken secondary antibody (1:5000, Life technologies, A-11039). Images were taken with an Olympus Fluoview FV1000 confocal microscope and processed with Fiji [30].

\section{Statistics}

All statistical analyses were performed using GraphPad Prism version 8.1.1 software. Exact sample sizes as well as description of sample collection, number of times the experiments were replicated and statistical measures and methods can be found in the figure legends. When appropriate, a Student's unpaired two-tailed $t$ test or a two-way ANOVA followed by an uncorrected Fisher's LSD multiple comparison test was used. Outliers were identified via the Grubbs' test and subsequently removed. Instances of outlier removal are detailed in the relevant figure legends. For the Kaplan-Meier survival analysis, a log-rank test was used. 


\section{Results}

\section{Muscle-specific KIf15 expression with a scAAV2/ 8-Spc5-12 viral vector}

To specifically induce Klf15 expression in skeletal muscle, we utilized a self-complementary adeno-associated virus serotype $2 / 8$ driven by the synthetic muscle-specific promoter Spc5-12 [27] (scAAV2/8-Spc5-12-Klf15, henceforth termed AAV8-Klfl5). This combination of AAV and promoter has previously been successfully used for gene delivery to muscles for treatment of the muscle disorder Duchenne muscular dystrophy (DMD) [31]. A control scAAV2/8-Spc5-12-GFP construct was also generated (henceforth termed AAV8-GFP).

We first examined the transduction ability of the AAV8GFP construct in differentiated $\mathrm{C} 2 \mathrm{C} 12$ myoblasts [28]. The cells were transduced with AAV8-GFP (multiplicity of infection (MOI) $1 \mathrm{E} 10^{5}$ ) for 3 days and assessed for GFP expression compared with untreated cells. Both flow cytometry and live imaging analyses confirm the abundant presence of GFP in AAV8-transduced cells (Fig. 1a, b). Differentiated C2C12s transduced with AAV8-Klf15 (MOI $\left.1 \mathrm{E} 10^{5}\right)$ demonstrate a significant increased expression of Klf15 mRNA compared with untreated cells (Fig. 1c).

To determine if our constructs would also be active at early time points in muscle of neonatal mice, we administered AAV8-GFP (1E11 vg/pup) to P0 WT pups via a facial vein intravenous injection [26]. Quadriceps muscles were harvested from injected and non-injected WT littermates at P2 and P7. P2 represents the presymptomatic age at which we have observed a significant downregulation of Klfl5 in the Taiwanese $S m n^{-1-} ;$ SMN2 SMA mice $[18,24]$ while P7 is considered a late symptomatic time point. qPCR analysis shows a significant upregulation of GFP expression at both P2 and P7, albeit variable between animals, with increased levels at the later time point (Fig. 1d). Immunohistochemistry of $\mathrm{P} 2$ and $\mathrm{P} 7$ quadriceps also reveals a time-dependent increased expression of GFP in AAV8-treated animals compared with untreated littermates (Fig. 1e). Combined, our experiments in $\mathrm{C} 2 \mathrm{C} 12 \mathrm{~s}$ and WT mice demonstrate the ability of our viral vectors to induce Klf15 and GFP expression in differentiated skeletal muscle.

\section{Neonatal administration of AAV8-KIf15 to severe SMA mice does not improve weight or survival}

We next wanted to determine if increasing early postnatal Klf15 expression in the $S m n^{-1-}$;SMN2 SMA mice would influence disease progression. However, administration of $1 \mathrm{E} 11 \mathrm{vg} / \mathrm{pup}$ of AAV8-Klf15 to P0 Smn ${ }^{-1-} ; S M N 2$ mice and $S m n^{+-} ; S M N 2$ control littermates was in fact toxic (spontaneous death without any impact on weight) to both genotypes (data not shown). Seeing as this dose was not harmful with AAV8-GFP, the adverse effects are most likely due to the supraphysiological levels of Klf15. We therefore reduced the AAV8-GFP and AAV8-Klf15 dose to $2 \mathrm{E} 10 \mathrm{vg} / \mathrm{pup}$ for subsequent administrations, which still allowed for an age-dependent increased expression of GFP (Fig. 2a) and Klf15 (Fig. 2b), albeit with some variability between animals, in quadriceps of $\mathrm{P} 2$ and $\mathrm{P} 7 \mathrm{Smn}^{+-}$;SMN2 and $S m n^{-1-}$;SMN2 mice.

In terms of effects on disease progression, we found that AAV8-Klf15-treated $\mathrm{Smn}^{-l-}$;SMN2 mice survived longer than untreated $S m n^{-l-} ; S M N 2$ (Fig. 2c). However, AAV8GFP-treated $\mathrm{Smn}^{-1-} ; S M N 2$ mice also display a moderately improved lifespan (Fig. 2c), suggesting that the AAV construct itself has some beneficial physiological impact. Interestingly, we also observed that $S m n^{-l-} ; S M N 2$ mice that received the AAV8-GFP weighed slightly more than AAV8-Klf15-treated and untreated $S m n^{-l-}$;SMN2 (Fig. 2d).

We found no significant differences between the survival of untreated, AAV8-GFP-treated and AAV8-Klf15-treated $\mathrm{Smn}^{+/-}$;SMN2 mice (Fig. 2e), although a small number of spontaneous deaths occurred in all cohorts. Similar to what we observed in the $S m n^{-1-} ; S M N 2$ mice, AAV8-GFPtreated $\mathrm{Smn}^{+/-} ; S M N 2$ mice weighed slightly more than untreated and AAV8-Klf15-treated $\mathrm{Smn}^{+/-} ; S M N 2$ mice during the $\mathrm{P} 0-\mathrm{P} 21$ preweaning phase (Fig. 2f). However, this increased weight was not maintained post-weaning (Fig. 2g). We did observe a small but significant increase in weight of AAV8-Klf15-treated $\mathrm{Smn}^{+-}$;SMN2 mice at 6 months of age. Thus, administering a perinatal injection of AAV8-Klf15 at a dose of 2E10 vg/pup significantly increases Klf15 expression in skeletal muscle without having overt adverse or beneficial effects on survival. Of note, a smaller AAV8-Klf15 dose of 1E10 vg/pup was also assessed but did not display significant survival benefits compared with the 2E10 vg/pup dose (Supplementary Fig. 1).

\section{Neonatal administration of AAV8-KIf15 to intermediate SMA mice slightly improves survival}

Due to the severe and rapid disease progression in the $\mathrm{Smn}^{-1-}$; $S M N 2$ mice, they respond less favorably to non-SMN treatment strategies compared with the milder intermediate $S m n^{2 B /-}$ mouse model [18, 25, 32, 33]. We therefore proceeded to evaluate the effect of AAV8-Klf15 in Smn ${ }^{2 B /-}$ mice and $\mathrm{Sm}^{2 \mathrm{~B} / 4}$ control littermates, following the same dosing regimen as in the severe SMA mice. Similar to what was observed in the $S m n^{-1-} ; S M N 2$ mice, AAV8-GFP-treated $S m n^{2 B /-}$ mice also demonstrated a small but significant increase in survival compared with untreated $S m n^{2 B /-}$ mice (Fig. 3a). Interestingly, AAV8-Klf15-treated $S m n^{2 B /-}$ mice had a significantly increased lifespan compared with both untreated and AAV8-GFP-treated 
A

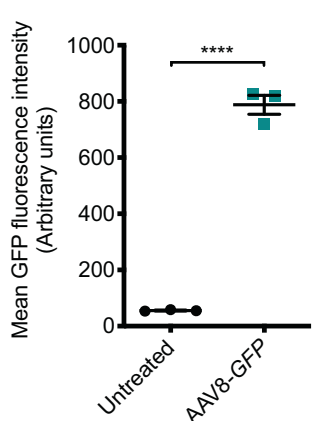

C

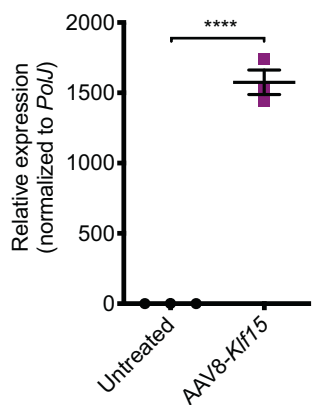

E
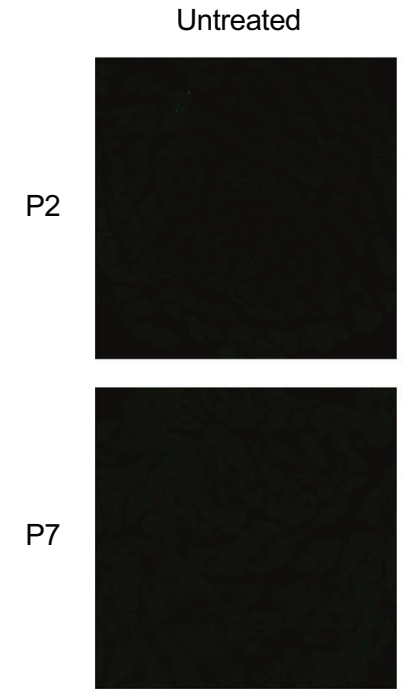

D
B
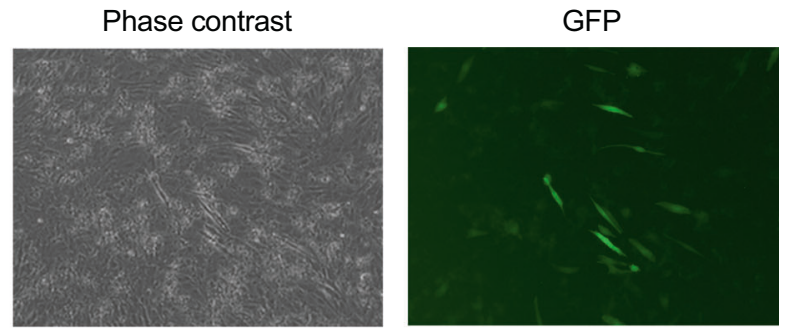

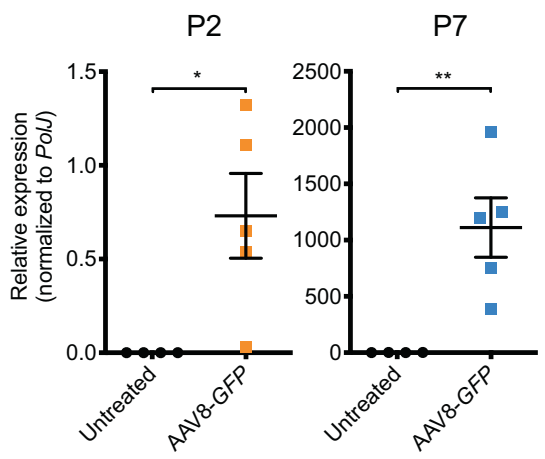

AAV8-GFP
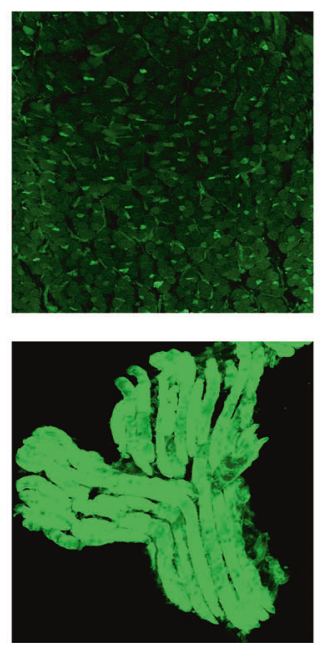

Fig. 1 Muscle-enhanced expression with the scAAV2/8-Spc5-12Klf15 and -GFP (AAV8-Klf15 and AAV8-GFP) viral vectors. a Mean GFP fluorescence intensity (arbitrary units) determined by flow cytometry analysis in differentiated $\mathrm{C} 2 \mathrm{C} 12 \mathrm{~s}$, untreated or transduced with AAV8-GFP (MOI $1 \mathrm{E} 10^{5}$ ) for 3 days. Data are scatter plot and mean $\pm \mathrm{SEM}, n=3$ wells per experimental group, unpaired $t$-test, $* * * * p<0.0001$. b Representative images (phase contrast and GFP fluorescent signal) of differentiated $\mathrm{C} 2 \mathrm{C} 12$ cells 3 days posttransduction with AAV8-GFP (MOI $1 \mathrm{E} 10^{5}$ ). c qPCR analysis of Klf15 mRNA expression in differentiated $\mathrm{C} 2 \mathrm{C} 12 \mathrm{~s} 3$ days posttransduction with AAV8-Klf15 (MOI 1E10 ${ }^{5}$ ) compared with untreated

Smn ${ }^{2 B /-}$ mice (Fig. 3a). While AAV8-Klf15 did not influence the weight of $S m n^{2 B /-}$ mice compared with untreated and AAV8-GFP-treated animals during the nursing period cells. Data are scatter plot and mean \pm SEM, $n=3$ wells per experimental group, unpaired $t$-test, $* * * * p<0.0001$. d qPCR analysis of GFP mRNA expression in quadriceps muscles of postnatal day (P) 2 and P7 wild-type (WT) animals that received a facial vein intravenous injection of AAV8-GFP (1E11 vg/pup) at P0 compared with untreated WT mice. Data are scatter plot and mean \pm SEM, $n=4-5$ animals per experimental group, unpaired $t$-test, $* p=0.028(\mathrm{P} 2),{ }^{*} * p=0.0076$ (P7). e Representative images of cross-sections (P2) and longitudinal sections (P7) of quadriceps immunostained for GFP from P2 and P7 untreated WT animals and WT mice that received a facial vein intravenous injection of AAV8-GFP (1E11 vg/pup) at P0.

(up to P21), post-weaned mice demonstrate a growthdependent gain in weight (Fig. 3b). However, that weight gain did not ultimately prevent an early death. Interestingly, 


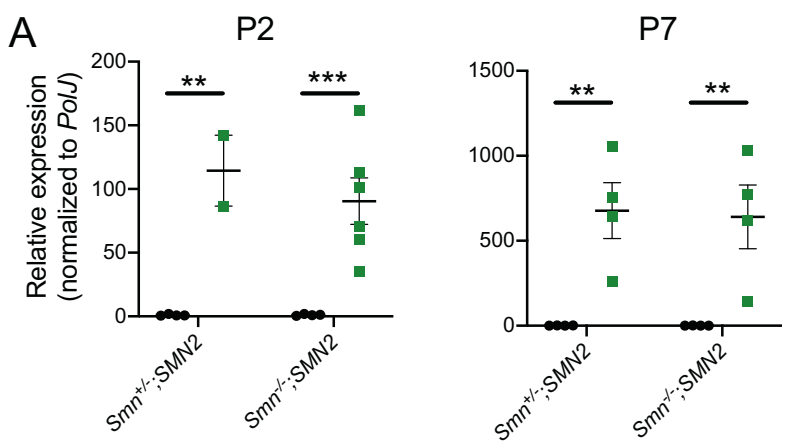

B

P2

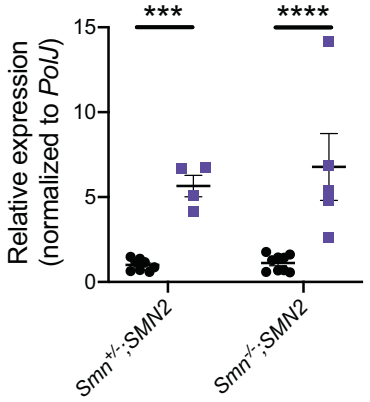

P7

- Untreated

- AAV8-GFP

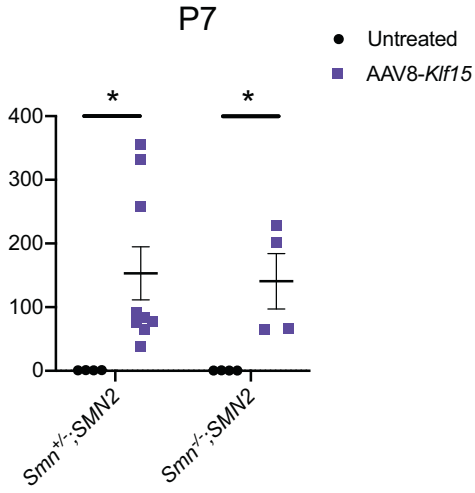

C

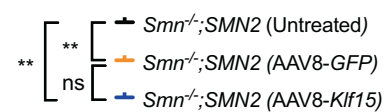

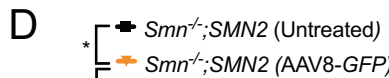

$=$ Smn - - SMN2 (AAV8-GFP)
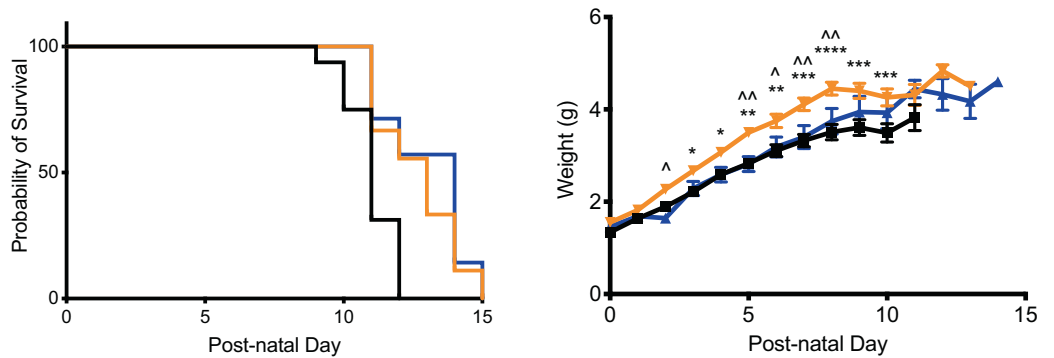

E

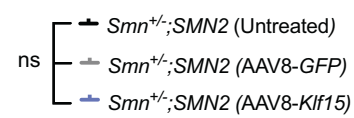

F

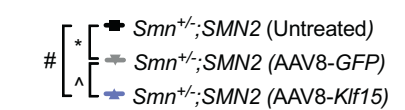

G
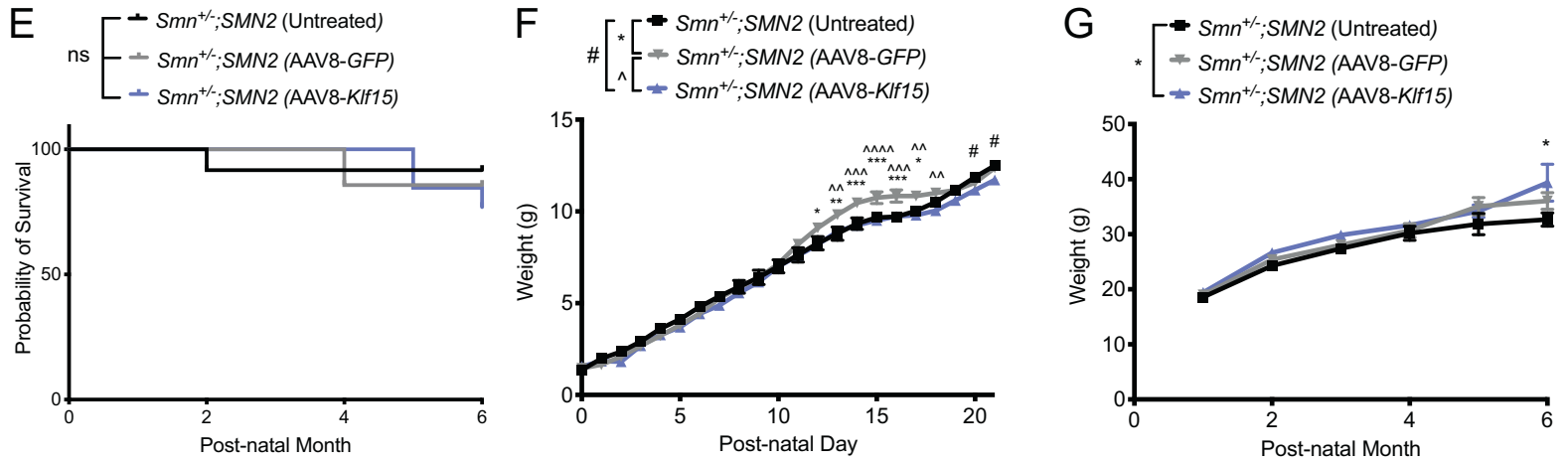

AAV8-GFP-treated $S m n^{2 B /-}$ mice were significantly heavier than untreated and AAV8-Klfl5-treated $S m n^{2 B /-}$ mice (Fig. 3b) between P13 and 21, again similar to what we found in the
$S m n^{-1-}$;SMN2 mice. We did not observe any effects of AAV8GFP or AAV8-Klf15 on the weights of preweaned $S m n^{2 B /-}$ mice compared with untreated animals (Fig. 3c). Thus, overall, 
Fig. 2 Perinatal administration of AAV8-Klf15 does not improve weight or survival of $\mathbf{S m n}^{-/-}$;SMN2 SMA mice. Postnatal day (P) 0 $S m n^{-1-} ;$ SMN2 SMA mice and control littermates were either untreated or received a single facial vein intravenous injection of AAV8-GFP or AAV8-Klf15 (2E10 vg/pup). a qPCR analysis of GFP mRNA expression in quadriceps of P2 and P7 untreated and AAV8-GFPtreated $\mathrm{Smn}^{+-} ;$;SMN2 and $\mathrm{Smn}^{-1-} ; \mathrm{SMN} 2$ mice. Data are scatter plot and mean \pm SEM, $n=2-6$ animals per experimental group, two-way ANOVA, $* * p<0.01, * * * p<0.001$. b qPCR analysis of Klf15 mRNA expression in quadriceps of P2 and P7 untreated and AAV8-Klf15treated $\mathrm{Smn}^{+/-} ;$SMN2 and $\mathrm{Smn}^{-1-}$;SMN2 mice. Data are scatter plot and mean \pm SEM, $n=4-9$ animals per experimental group, two-way ANOVA, $* p<0.05, * * * p<0.001, * * * * p<0.0001$. One outlier identified by the Grubbs' test was removed from the P7 $S m n^{-1-} ; S M N 2$ group. c Survival curves of untreated $(n=16)$, AAV8-GFP-treated $(n=9)$, and AAV8-Klf15-treated $(n=7) S m n^{-/-} ; S M N 2$ mice. Data are Kaplan-Meier survival curves, log-rank Mantel-Cox test, ns = not significant, $* * p=0.0034$ (untreated vs AAV8-GFP), $* * p=0.0048$ (untreated vs AAV8-Klf15). d Daily weights of untreated $(n=16)$, AAV8-GFP-treated $(n=9)$ and AAV8-Klf15-treated $(n=7) S m n^{-l-}$; $S M N 2$ mice. Data are mean \pm SEM, two-way ANOVA, $* / \wedge p<0.05$, $* * / \wedge \wedge p<0.01, * * * p<0.001, * * * * p<0.0001$. e Survival curves of untreated $(n=12)$, AAV8-GFP-treated $(n=7)$ and AAV8-Klf15treated $(n=13) \mathrm{Smn}^{+/-}$;SMN2 mice. Data are Kaplan-Meier survival curves, log-rank Mantel-Cox test, $\mathrm{ns}=$ not significant. f Daily weights of untreated $(n=11)$, AAV8-GFP-treated $(n=8)$ and AAV8-Klf15treated $(n=12) \mathrm{Smn}^{+/-} ; S M N 2$ mice. Data are mean \pm SEM, two-way ANOVA, $* / \wedge / \# p<0.05, * * / \wedge \wedge p<0.01, * * * / \wedge \wedge \wedge p<0.001,{ }^{\wedge \wedge \wedge \wedge} p<$ 0.0001. g Monthly weights of untreated $(n=11)$, AAV8-GFP-treated $(n=7)$ and AAV8-Klf15-treated $(n=13) S m n^{+/-} ; S M N 2$ mice. Data are mean \pm SEM, two-way ANOVA, ${ }^{*} p<0.05$.

the AAV8-Klf15 was slightly more beneficial in the intermediate $S m n^{2 B I-}$ SMA mouse model than the severe Taiwanese $\mathrm{Smn}^{-1-}$;SMN2 mice.

\section{The AAV8-Spc5-12 construct also induces expression in heart and liver}

While the synthetic Spc5-12 promoter has been used for its enhanced activity in skeletal muscle [27, 31], we wanted to determine if our AAV8 delivery system also induced expression in heart and liver, tissues in which Klf15 also plays key roles $[34,35]$. We indeed observed significant increased expression of GFP and Klf15 mRNA in the livers of both P2 and P7 AAV8-GFP- (Fig. 4a) and AAV8-Klf15treated (Fig. 4c) $\mathrm{Smn}^{+-}$;SMN2 and $\mathrm{Smn}^{-l-} ; S M N 2$ mice compared with untreated animals. This increase was $\sim 15$ times $(G F P)$ and $\sim 10$ times (Klfl5) higher than in skeletal muscle for both time points. Similarly, we find a significant upregulation of GFP and Klf15 mRNA in the hearts of AAV8-GFP- (Fig. 4c) and AAV8-Klf15-treated (Fig. 4d) P2 and P7 Smn ${ }^{+-} ;$SMN2 and $S m n^{-1-} ; S M N 2$ mice compared with untreated animals. Surprisingly, the increase in heart was $\sim 40$ times $(G F P)$ and $\sim 50$ times (Klf15) higher at $\mathrm{P} 2$, while it was $\sim 70$ times $(G F P$ ) and $\sim 10$ times (Klf15) higher at P7 compared with skeletal muscle at those respective time points. Furthermore, qPCR analysis of

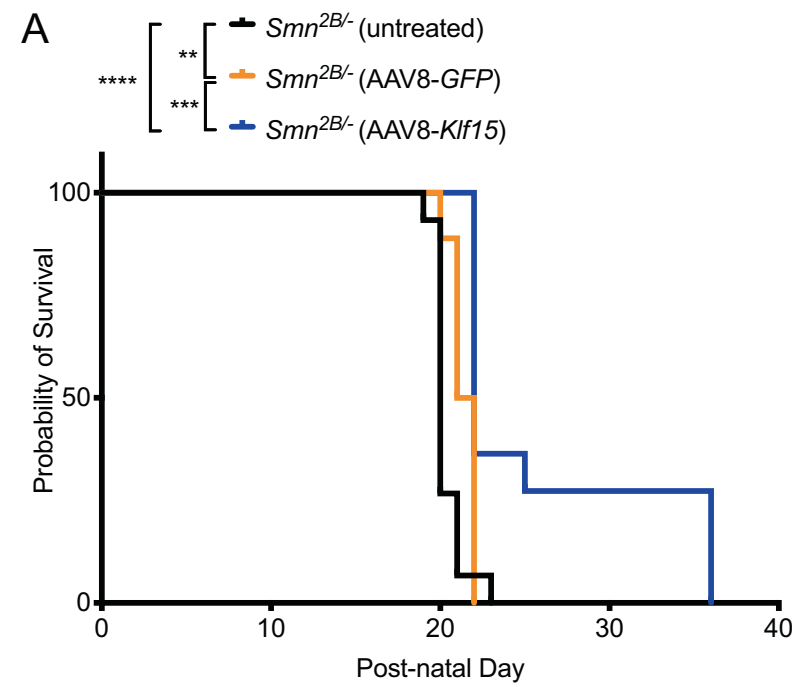

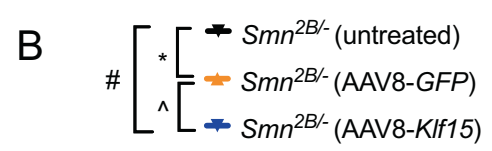
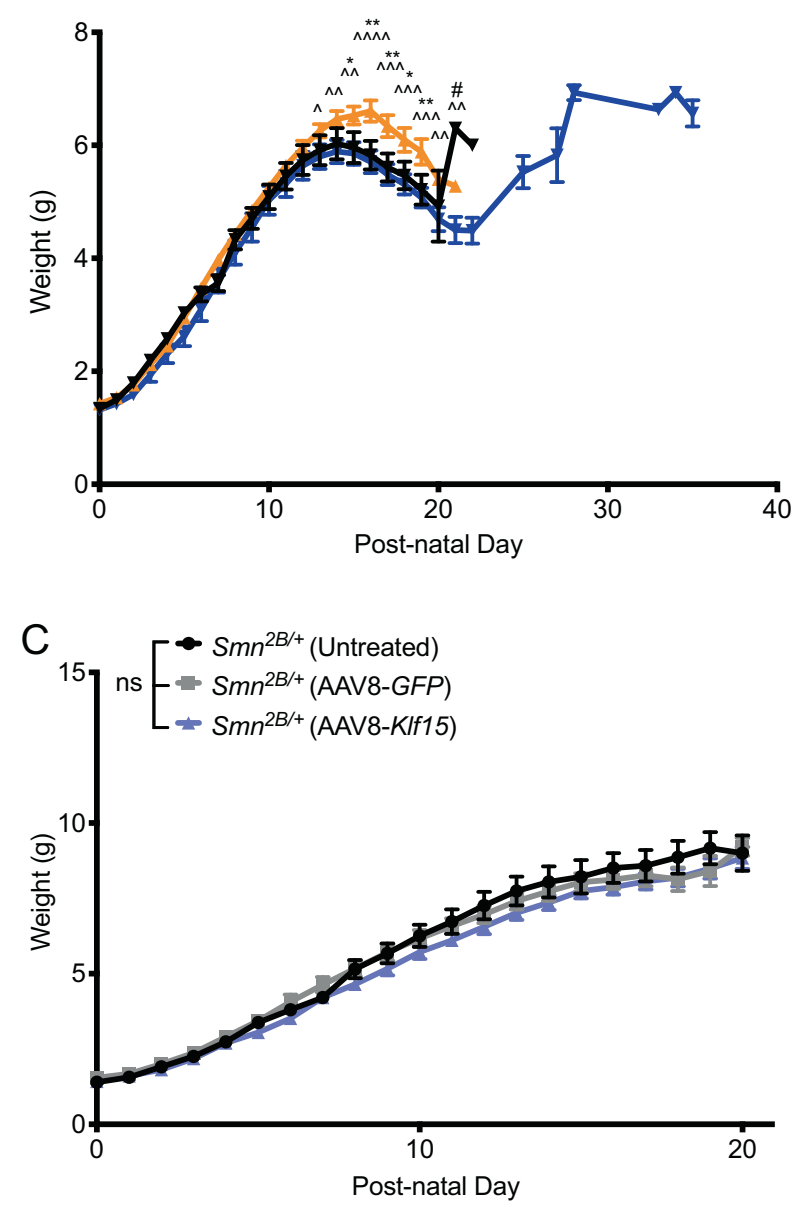

AAV8 content in respective tissues, using capsid-specific primers, indeed demonstrates increased AAV8 presence in heart and liver compared with muscle, particularly at P2 
Fig. 3 Perinatal administration of AAV8-Klf15 slightly increases survival in $\mathbf{S m n}^{\mathbf{2 B /} /-}$ SMA mice. Postnatal day (P) $0 \mathrm{Smn}^{2 B /-}$ SMA mice and control littermates were either untreated or received a single facial vein intravenous injection of AAV8-GFP or AAV8-Klf15 (2E10 $\mathrm{vg} /$ pup). a Survival curves of untreated $(n=15)$, AAV8-GFP-treated $(n=18)$ and AAV8-Klf15-treated $(n=11) S m n^{2 B /-}$ mice. Data are Kaplan-Meier survival curves, log-rank Mantel-Cox test, ${ }^{* *} p=$ 0.0021 (untreated vs AAV8-GFP), **** $p<0.0001$ (untreated vs AAV8-Klf15), $* * * p=0.0008$ (AAV8-GFP vs AAV8-Klf15). b Daily weights of untreated $(n=18)$, AAV8-GFP-treated $(n=18)$ and AAV8-Klf15-treated $(n=11) S m n^{2 B /-}$ mice. Data are mean \pm SEM, two-way ANOVA, $* / \wedge / \# p<0.05, \quad * * / \wedge \wedge p<0.01, \quad \wedge^{\wedge \wedge \wedge} p<0.001$, $\wedge^{\wedge \wedge \wedge} p<0.0001$. c Daily weights of untreated $(n=11)$, AAV8-GFPtreated $(n=9)$, and AAV8-Klf15-treated $(n=16) S m n^{2 B /+}$ mice. Data are mean \pm SEM, two-way ANOVA, ns $=$ not significant.

(Supplementary Fig. 2). Of note, there was some variability between animals within the same experimental group. Therefore, our results demonstrate that the activity of the AAV8-Spc5-12 vector is not exclusive to skeletal muscle and in fact, appears to display a greater tropism for nonskeletal muscle tissues when administered intravenously in newborn animals.

\section{Discussion}

We have recently demonstrated that Klf15 expression is significantly downregulated in muscle of presymptomatic SMA mice and that upregulating Klf15 expression via genetic (transgenic muscle-specific expression) or pharmacological (prednisolone) approaches results in improved disease phenotypes [18]. Here, we evaluated the impact of specifically upregulating Klf15 in skeletal muscle in perinatal mice by driving its expression via an AAV8-Spc5-12 vector. We find that while neonatal administration of the AAV8-Klf15 construct leads to significant increased levels of Klf15 in muscle, this has no overt effect on survival or weight gain in the severe Taiwanese SMA mice, while we observe a small improvement in the lifespan of the intermediate $S m n^{2 B /-}$ mice.

The lack of significant impact of AAV8-Klf15 in severe SMA mice may be due to several compounding factors. While the levels of Klf15 expression achieved with AAV8Klf15 are similar to the levels observed in transgenic SMA mice overexpressing muscle-specific Klf15 at P2 ( 5-10 fold greater than control littermates), the amounts measured in P7 AAV8-Klf15-treated animals are significantly greater than the transgenic mice ( $\sim 65-740$ fold greater and $\sim 30$ fold greater, respectively, compared with control littermates) (Fig. 2) [18]. As Klf15 can display both atrophy-inducing [36] and ergogenic [19] properties in skeletal muscle in a dose-dependent manner [37], it is quite possible that the supraphysiological levels achieved with AAV8-Klf15 favor muscle wasting over growth. We also note significantly more variability in Klf15 levels in animals injected with the AAV8 construct compared with the transgenic mice (Fig. 2) [18], which are most likely due to differential injection efficiencies and/or vector spread and could influence physiological outcomes.

We have previously shown that administration of prednisolone to SMA mice also increases Klf15 levels in skeletal muscle of P2 presymptomatic animals ( 6 fold greater than untreated controls) [18]. However, this effect on Klf15 induction ceased at P7, specifically in SMA mice [18], suggesting that prednisolone-dependent benefits in symptomatic SMA mice may be due to KLF15independent effects and/or that prednisolone-dependent Klf15 increase in P7 animals may be limited by compensatory inhibitory mechanisms due to already significantly increased Klf15 levels in symptomatic SMA mice compared with controls [18]. It is therefore possible that an optimal strategy would be to conditionally increase Klfl5 expression in presymptomatic stages only, which is not easily achieved as the kinetics of AAV-mediated overexpression require several days for efficient transgene activity. To achieve optimal expression at early presymptomatic postnatal stages may therefore require prenatal delivery.

While our AAV8 construct was designed to overexpress Klf15 specifically in skeletal muscle, our analysis of heart and liver demonstrates significantly higher activity in these tissues (Fig. 4 and Supplementary Fig. 2). Tropism of the AAV8 construct and the Spc5-12 promoter, alone and in combination, has indeed previously been reported in both the heart and liver of adult mice [31,38]. Our data support that this is also the case in neonatal mice. KLF15 has well described roles in heart and liver [34, 35], suggesting that its increased expression via our AAV8 construct, most likely also impacts the function of these tissues. SMA mice display several heart and liver pathologies [39, 40], suggesting that aberrant KLF15 levels may have non-intended organspecific adverse effects, which most likely explain the spontaneous deaths observed in our mice treated with the high vector dose. Furthermore, we have previously reported increased levels of Klfl5 in the liver and heart of symptomatic mice [18], which most likely reflect a pathological response. However, as only one preparation of AAV8-Klf15 was used throughout this study, its toxicity may have been due to batch-specific impurities [41]. However, seeing as the toxicity of AAV8-Klf15 was dose-dependent in both compromised SMA mice and healthy littermates and not observed in parallel experiments with AAV8-GFP, it is most likely that the adverse effects were directly linked to the supraphysiological levels of Klf15 in several key metabolic tissues such as muscle, heart and liver. Thus, the dose-dependent effects of AAV8-Spc5-12-mediated Klf15 upregulation are probably due to a complex interplay 
Fig. 4 Perinatal

administration of the AAV8Spc5-12 construct induces high expression in liver and heart. Postnatal day (P) 0 $\mathrm{Smn}^{-1-}$;SMN2 SMA mice and control littermates were either untreated or received a single facial vein intravenous injection of AAV8-GFP or AAV8-Klf15 (2E10 vg/pup). qPCR analysis of GFP (a) and Klf15 (b) mRNA expression in quadriceps muscles of P2 and P7 untreated and AAV8-treated $\mathrm{Smn}^{+/-}$; SMN2 and $\mathrm{Smn}^{-1-}$;SMN2 mice. Data are scatter plot and mean \pm SEM, $n=2-6$ animals per experimental group, two-way ANOVA, $* * p<0.01, * * * p<$ $0.001, * * * * p<0.0001$. One outlier identified by the Grubbs' test was removed from the P7 liver AAV8-Klf15 $\mathrm{Smn}^{-/-}$; $S M N 2$ group. qPCR analysis of GFP (c) and Klf15 (d) mRNA expression in heart of $\mathrm{P} 2$ and $\mathrm{P} 7$ untreated and AAV8-treated $\mathrm{Smn}^{+/-}$;SMN2 and $\mathrm{Smn}^{-1-}$; $S M N 2$ mice. Data are scatter plot and mean $\pm \mathrm{SEM}, n=2-8$ animals per experimental group, two-way ANOVA, $* * p<0.01$, $* * p<0.01, * * * p<0.001$ $* * * * p<0.0001$. One outlier identified by the Grubbs' test was removed from the P2 heart untreated $\mathrm{Smn}^{-1-}$;SMN2 group.
A

P2 Liver

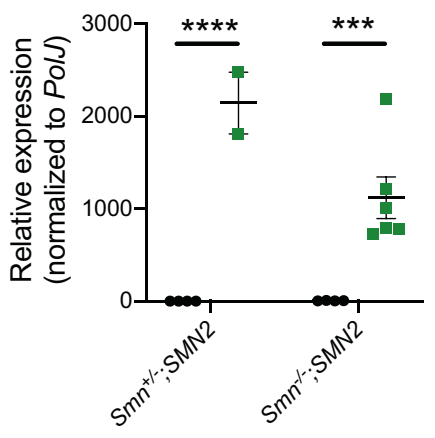

B

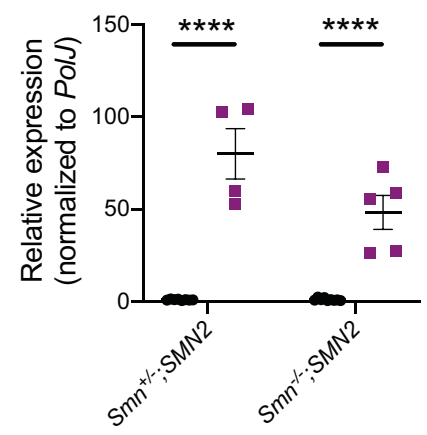

C P2 Heart
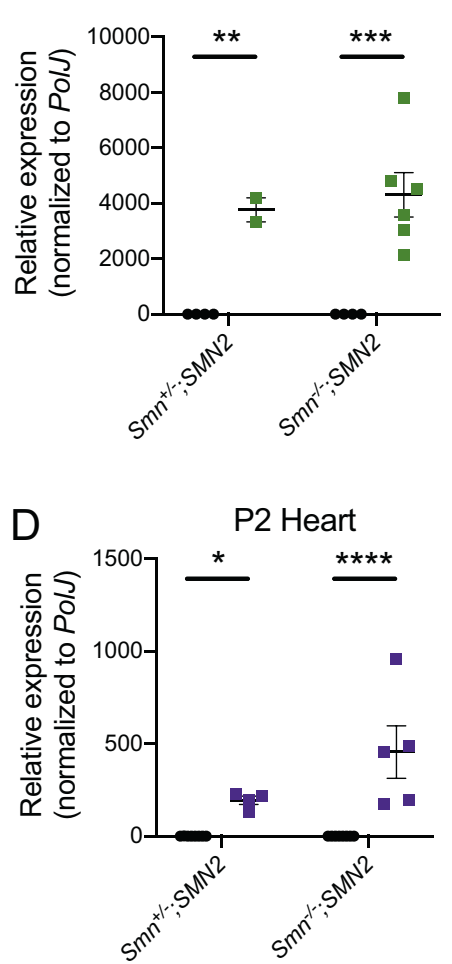

P2 Heart

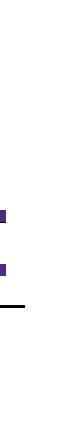

P7 Liver

- Untreated

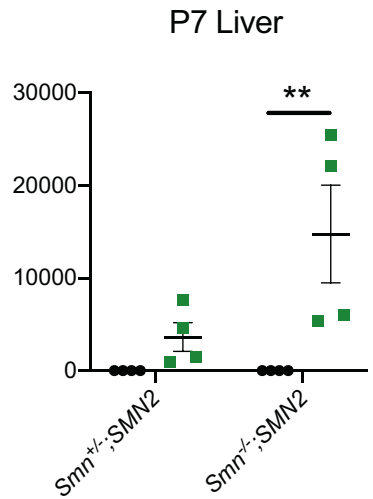

- AAV8-GFP

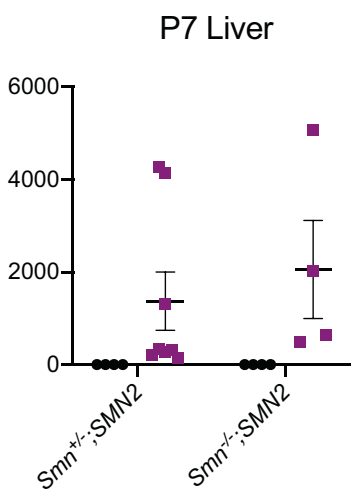

Untreated

- AAV8-KIf15

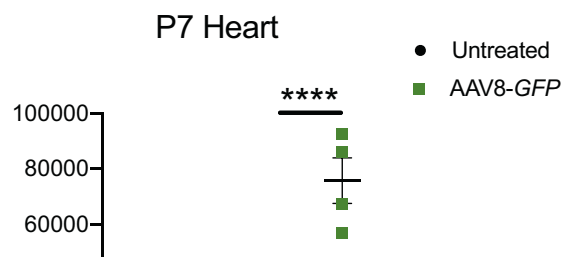

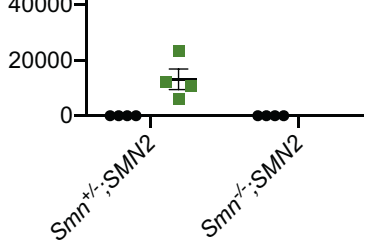

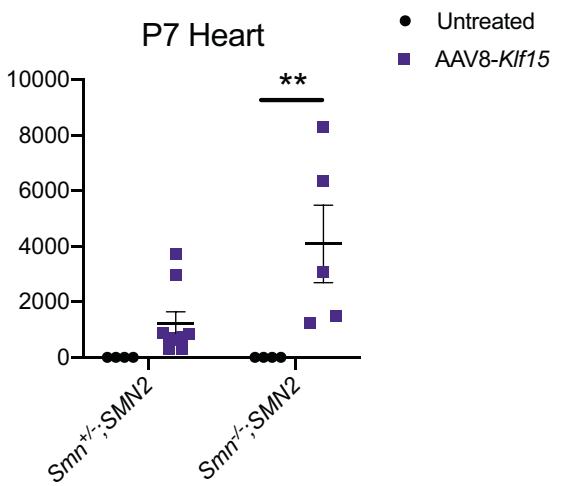

between tissue- and age-dependent beneficial and adverse signaling pathways that should be considered and evaluated in future investigations.
Surprisingly, the AAV8-GFP construct also demonstrated some non-negligible effects on disease phenotypes of SMA mice (Fig. 3). While we cannot be certain as to why 
that is, we speculate that it may be related to possible effects on the immune system, similarly to previous reports for AAV8 vectors [42, 43]. Seeing as SMA mice have an altered immune response $[44,45]$ and that inflammation can display both protective and adverse systemic properties [46], including within the CNS [47] and muscle [48], it is possible that an activated immune response results in acute and/or intermittent benefits in AAV8-treated SMA animals.

In summary, the limited impact of AAV8-Klf15 administration in SMA mice might be explained by several experimental conditions that most likely reduced our ability to increase Klf15 specifically in skeletal muscle at physiological levels and with the optimal timing, without influencing the function of other tissues and systems. In the experimental paradigms tested here, the positive, albeit small, effect on survival and weight was restricted to the milder $\mathrm{Smn}^{2 \mathrm{Bl}-}$ SMA mouse model. Future investigations will require endeavors to further optimize a muscle-specific construct by either reconfiguring the AAV/promoter combination and/or inhibiting its expression in non-skeletal muscle tissues.

Acknowledgements NA, DH, RJYM, and MB were supported by the UK SMA Research Consortium SMA Trust UK grant. SMH is funded by the MRC DPFS (MR/R025312/1). SK was supported by an ERASMUS grant, and PC received financial support from the Deutsche Muskelstiftung.

Author contributions Conceptualization: NA, RJYM, and MB. Methodology: NA, DH, RJYM, and MB. Formal Analysis: NA, AK, SK, and MB. Investigation: NA, AK, SK, and MB. Resources: SMH, MJAW, RJYM, and MB; Writing-Original draft: NA and MB. Writing-Review and Editing: NA, DH, AK, SK, PC, RJYM, and MB. Visualization: NA, AK, and MB. Supervision: PC, SMH, MJAW, and MB. Project Administration: RJYM and MB. Funding Acquisition: SHM, MJAW, RJYM, and MB.

\section{Compliance with ethical standards}

Conflict of interest RJYM was Editor-in-Chief of Gene Therapy. All other authors have no conflicts of interest to declare.

Publisher's note Springer Nature remains neutral with regard to jurisdictional claims in published maps and institutional affiliations.

Open Access This article is licensed under a Creative Commons Attribution 4.0 International License, which permits use, sharing, adaptation, distribution and reproduction in any medium or format, as long as you give appropriate credit to the original author(s) and the source, provide a link to the Creative Commons license, and indicate if changes were made. The images or other third party material in this article are included in the article's Creative Commons license, unless indicated otherwise in a credit line to the material. If material is not included in the article's Creative Commons license and your intended use is not permitted by statutory regulation or exceeds the permitted use, you will need to obtain permission directly from the copyright holder. To view a copy of this license, visit http://creativecommons. org/licenses/by/4.0/.

\section{References}

1. Crawford TO, Pardo CA. The neurobiology of childhood spinal muscular atrophy. Neurobiol Dis. 1996;3:97-110.

2. Pearn J. Classification of spinal muscular atrophies. Lancet. 1980;1:919-22.

3. Lefebvre S, Bürglen L, Reboullet S, Clermont O, Burlet P, Viollet $\mathrm{L}$, et al. Identification and characterization of a spinal muscular atrophy-determining gene. Cell. 1995;80:155-65.

4. Schrank B, Götz R, Gunnersen JM, Ure JM, Toyka KV, Smith AG, et al. Inactivation of the survival motor neuron gene, a candidate gene for human spinal muscular atrophy, leads to massive cell death in early mouse embryos. Proc Natl Acad Sci USA. 1997;94:9920-5.

5. Monani UR, Lorson CL, Parsons DW, Prior TW, Androphy EJ, Burghes $\mathrm{AH}$, et al. A single nucleotide difference that alters splicing patterns distinguishes the SMA gene SMN1 from the copy gene SMN2. Hum Mol Genet. 1999;8:1177-83.

6. Lorson CL, Hahnen E, Androphy EJ, Wirth B. A single nucleotide in the SMN gene regulates splicing and is responsible for spinal muscular atrophy. Proc Natl Acad Sci USA. 1999;96:6307-11.

7. Lefebvre S, Burlet P, Liu Q, Bertrandy S, Clermont O, Munnich A, et al. Correlation between severity and SMN protein level in spinal muscular atrophy. Nat Genet. 1997;16:265-9.

8. Chiriboga CA. Nusinersen for the treatment of spinal muscular atrophy. Expert Rev Neurother. 2017;17:955-62.

9. Singh NN, Lee BM, DiDonato CJ, Singh RN. Mechanistic principles of antisense targets for the treatment of spinal muscular atrophy. Future Med Chem. 2015;7:1793-808.

10. Hoy SM. Onasemnogene abeparvovec: first global approval. Drugs. 2019;79:1255-62.

11. Wood MJA, Talbot K, Bowerman M. Spinal muscular atrophy: antisense oligonucleotide therapy opens the door to an integrated therapeutic landscape. Hum Mol Genet. 2017;26:R151-9.

12. Bowerman M, Becker CG, Yáñez-Muñoz RJ, Ning K, Wood MJA, Gillingwater $\mathrm{TH}$, et al. Therapeutic strategies for spinal muscular atrophy: SMN and beyond. Dis Model Mech. 2017;10:943-54.

13. Gidaro T, Servais L. Nusinersen treatment of spinal muscular atrophy: current knowledge and existing gaps. Dev Med Child Neurol. 2019;61:19-24.

14. Lipnick SL, Agniel DM, Aggarwal R, Makhortova NR, Finlayson $\mathrm{SG}$, Brocato A, et al. Systemic nature of spinal muscular atrophy revealed by studying insurance claims. PLoS ONE. 2019;14: e0213680.

15. Bowerman M. Funding for spinal muscular atrophy research must continue. Future Neurol. 2019;14:FNL10.

16. Boyer JG, Ferrier A, Kothary R. More than a bystander: the contributions of intrinsic skeletal muscle defects in motor neuron diseases. Front Physiol. 2013;4:356.

17. Bowerman M, Murray LM, Scamps F, Schneider BL, Kothary R, Raoul C. Pathogenic commonalities between spinal muscular atrophy and amyotrophic lateral sclerosis: converging roads to therapeutic development. Eur J Med Genet. 2018;61:685-98.

18. Walter LM, Deguise M-O, Meijboom KE, Betts CA, Ahlskog N, van Westering TLE, et al. Interventions Targeting GlucocorticoidKrüppel-like Factor 15-Branched-Chain amino acid signaling improve disease phenotypes in spinal muscular atrophy mice. EBioMedicine. 2018;31:226-42.

19. Morrison-Nozik A, Anand P, Zhu H, Duan Q, Sabeh M, Prosdocimo DA, et al. Glucocorticoids enhance muscle endurance and ameliorate Duchenne muscular dystrophy through a defined metabolic program. Proc Natl Acad Sci USA. 2015;112:E6780-9.

20. Haldar SM, Jeyaraj D, Anand P, Zhu H, Lu Y, Prosdocimo DA, et al. Kruppel-like factor 15 regulates skeletal muscle lipid flux 
and exercise adaptation. Proc Natl Acad Sci USA. 2012;109: 6739-44.

21. Gray S, Wang B, Orihuela Y, Hong E-G, Fisch S, Haldar S, et al. Regulation of gluconeogenesis by Krüppel-like factor 15. Cell Metab. 2007;5:305-12.

22. Jeyaraj D, Scheer FAJL, Ripperger JA, Haldar SM, Lu Y, Prosdocimo DA, et al. Klf15 orchestrates circadian nitrogen homeostasis. Cell Metab. 2012;15:311-23.

23. El-Brolosy MA, Stainier DYR. Genetic compensation: a phenomenon in search of mechanisms. PLOS Genet. 2017;13: e1006780.

24. Hsieh-Li HM, Chang JG, Jong YJ, Wu MH, Wang NM, Tsai CH, et al. A mouse model for spinal muscular atrophy. Nat Genet. 2000;24:66-70.

25. Bowerman M, Murray LM, Beauvais A, Pinheiro B, Kothary R. A critical SMN threshold in mice dictates onset of an intermediate spinal muscular atrophy phenotype associated with a distinct neuromuscular junction pathology. Neuromuscul Disord NMD. 2012;22:263-76.

26. Gombash Lampe SE, Kaspar BK, Foust KD. Intravenous injections in neonatal mice. J Vis Exp. 2014;93:e52037. https://doi.org/ $10.3791 / 52037$.

27. Li X, Eastman EM, Schwartz RJ, Draghia-Akli R. Synthetic muscle promoters: activities exceeding naturally occurring regulatory sequences. Nat Biotechnol. 1999;17:241.

28. Blau HM, Pavlath GK, Hardeman EC, Chiu CP, Silberstein L, Webster SG, et al. Plasticity of the differentiated state. Science. 1985;230:758-66.

29. Radonić A, Thulke S, Mackay IM, Landt O, Siegert W, Nitsche A. Guideline to reference gene selection for quantitative real-time PCR. Biochem Biophys Res Commun. 2004;313:856-62.

30. Schindelin J, Arganda-Carreras I, Frise E, Kaynig V, Longair M, Pietzsch T, et al. Fiji: an open-source platform for biologicalimage analysis. Nat Methods. 2012;9:676-82.

31. Foster H, Sharp PS, Athanasopoulos T, Trollet C, Graham IR, Foster $\mathrm{K}$, et al. Codon and mRNA sequence optimization of microdystrophin transgenes improves expression and physiological outcome in dystrophic mdx mice following AAV2/8 gene transfer. Mol Ther J Am Soc Gene Ther. 2008;16:1825-32.

32. Kaifer KA, Villalón E, Osman EY, Glascock JJ, Arnold LL, Cornelison DDW, et al. Plastin-3 extends survival and reduces severity in mouse models of spinal muscular atrophy. JCI Insight. 2017;2:e89970.

33. Osman EY, Rietz A, Kline RA, Cherry JJ, Hodgetts KJ, Lorson $\mathrm{CL}$, et al. Intraperitoneal delivery of a novel drug-like compound improves disease severity in severe and intermediate mouse models of spinal muscular atrophy. Sci Rep. 2019;9:1633.
34. Zhao Y, Song W, Wang L, Rane MJ, Han F, Cai L. Multiple roles of KLF15 in the heart: Underlying mechanisms and therapeutic implications. J Mol Cell Cardiol. 2019;129:193-6.

35. Takashima M, Ogawa W, Hayashi K, Inoue H, Kinoshita S, Okamoto Y, et al. Role of KLF15 in regulation of hepatic gluconeogenesis and metformin action. Diabetes. 2010;59:1608-15.

36. Hirata Y, Nomura K, Senga Y, Okada Y, Kobayashi K, Okamoto $\mathrm{S}$, et al. Hyperglycemia induces skeletal muscle atrophy via a WWP1/KLF15 axis. JCI Insight. 2019;4. https://doi.org/10.1172/ jci.insight.124952.

37. Quattrocelli M, Barefield DY, Warner JL, Vo AH, Hadhazy M, Earley JU, et al. Intermittent glucocorticoid steroid dosing enhances muscle repair without eliciting muscle atrophy. J Clin Invest. 2017; 127:2418-32.

38. Sands MS. AAV-mediated liver-directed gene therapy. Methods Mol Biol. 2011;807:141-57.

39. Wijngaarde CA, Blank AC, Stam M, Wadman RI, van den Berg LH, van der Pol WL. Cardiac pathology in spinal muscular atrophy: a systematic review. Orphanet J Rare Dis. 2017;12:67.

40. Szunyogova E, Zhou H, Maxwell GK, Powis RA, Francesco M, Gillingwater TH, et al. Survival Motor Neuron (SMN) protein is required for normal mouse liver development. Sci Rep. 2016; 6:34635.

41. Penaud-Budloo M, François A, Clément N, Ayuso E. Pharmacology of recombinant adeno-associated virus production. Mol Ther Methods Clin Dev. 2018;8:166-80.

42. Reichel FF, Dauletbekov DL, Klein R, Peters T, Ochakovski GA, Seitz IP, et al. AAV8 can induce innate and adaptive immune response in the primate eye. Mol Ther. 2017;25:2648-60.

43. Wang L, Calcedo R, Wang H, Bell P, Grant R, Vandenberghe LH, et al. The pleiotropic effects of natural AAV infections on liverdirected gene transfer in Macaques. Mol Ther. 2010;18:126-34.

44. Deguise M-O, Kothary R. New insights into SMA pathogenesis: immune dysfunction and neuroinflammation. Ann Clin Transl Neurol. 2017;4:522-30.

45. Deguise M-O, De Repentigny Y, McFall E, Auclair N, Sad S, Kothary R. Immune dysregulation may contribute to disease pathogenesis in spinal muscular atrophy mice. Hum Mol Genet. 2017;26:801-19.

46. Butterfield TA, Best TM, Merrick MA. The dual roles of neutrophils and macrophages in inflammation: a critical balance between tissue damage and repair. J Athl Train. 2006;41:457-65.

47. Hohlfeld R, Kerschensteiner M, Meinl E. Dual role of inflammation in CNS disease. Neurology. 2007;68:S58-63. discussion S91-96

48. Yang W, Hu P. Skeletal muscle regeneration is modulated by inflammation. J Orthop Transl. 2018;13:25-32. 